\title{
Chronic Kidney Disease and SGLT2 Inhibitors: A Review of the Evolving Treatment Landscape
}

\author{
Christian W. Mende
}

Received: October 18, 2021 / Accepted: November 10, 2021 / Published online: November 30, 2021

(C) The Author(s) 2021

\section{ABSTRACT}

There is currently an unmet need for effective treatment of chronic kidney disease (CKD) that slows disease progression, prevents development of end-stage kidney disease and cardiovascular disease, and prolongs survival of patients with CKD. In the last 20 years, the only agents to show a reduction in the risk of CKD progression in patients with and without type 2 diabetes (T2D) were angiotensin-converting enzyme inhibitors and angiotensin receptor blockers, but neither drug class has provided a decreased risk of all-cause mortality in patients with CKD and evidence for their use in patients with CKD without T2D is relatively limited. This review discusses the mechanisms underlying the progression of $\mathrm{CKD}$, its associated risk factors, and summarizes the potential therapeutic approaches for managing CKD. There is increasing evidence to support the role of sodium-glucose cotransporter 2 (SGLT2) inhibitor therapy in patients with CKD, including data from the designated kidney outcome trials in patients with T2D (CREDENCE) and in patients with or without T2D (DAPA-CKD). These studies showed a significant reduction in the risk of CKD progression with canagliflozin

\section{W. Mende $(\bowtie)$}

Department of Medicine, University of California-

San Diego, 6950 Fairway Rd, La Jolla, CA 92037, USA

e-mail: cmende4730@aol.com (in patients with T2D) or dapagliflozin (in patients with or without T2D), respectively, with DAPA-CKD being the first trial to show a reduced risk of all-cause mortality. On the basis of these data, individualized treatment with SGLT2 inhibitors represents a promising therapeutic option for patients with diabetic and nondiabetic CKD to slow disease progression.

\section{PLAIN LANGUAGE SUMMARY}

Chronic kidney disease is a common condition in which the ability of the kidneys to work correctly gradually decreases over time. It is a major risk factor for a number of other serious conditions, including cardiovascular disease and end-stage kidney disease, and for early death. Several treatments have been shown to reduce the risk of chronic kidney disease progressing (particularly in patients with type 2 diabetes), but there have been no treatments that slow chronic kidney disease progression, prevent the development of end-stage kidney disease and cardiovascular disease, and prolong survival. However, evidence is now accumulating to suggest that some drugs initially developed to treat other diseases may be potential treatments for chronic kidney disease. The sodium-glucose cotransporter 2 inhibitors, which are commonly used to lower blood sugar 
levels in people with type 2 diabetes, are examples of such drugs. Data from two studies of sodium-glucose cotransporter 2 inhibitorsthe CREDENCE study of canagliflozin in patients with chronic kidney disease and type 2 diabetes and the DAPA-CKD study of dapagliflozin in patients with chronic kidney disease with or without type 2 diabetes-have shown that these drugs reduce the risk of chronic kidney disease progression in these patients. More importantly, the DAPA-CKD study showed that patients with chronic kidney disease who were taking dapagliflozin had a reduced risk of death compared with placebo. These results show that sodium-glucose cotransporter 2 inhibitors are slowing the progression of chronic kidney disease and improve overall outcomes for properly selected patients.

Keywords: Chronic kidney disease; Disease progression; Sodium-glucose cotransporter 2 inhibitors

\section{Key Summary Points}

An unmet need exists for additional effective treatments of chronic kidney disease (CKD) that slow disease progression and prolong survival.

The pathogenesis of CKD progression involves multifactorial mechanisms that must be considered to effectively manage and slow disease progression.

The key risk factors underlying CKD progression, including hyperglycemia, hypertension, albuminuria, and obesity, should be addressed.

Emerging evidence suggests that sodium-glucose cotransporter 2 inhibitors, the mineralocorticoid receptor antagonist finerenone, and glucagon-like peptide 1 receptor agonists may also provide benefits for slowing CKD progression and improving outcomes in patients with CKD.

\section{INTRODUCTION}

Chronic kidney disease (CKD) is defined based on the following criteria present for more than 3 months: estimated glomerular filtration rate (eGFR) $<60 \mathrm{~mL} / \mathrm{min} / 1.73 \mathrm{~m}^{2}$ or markers of kidney damage (structural or functional abnormalities; one or more): albuminuria (urine albumin excretion rate $\geq 30 \mathrm{mg} / 24 \mathrm{~h}$; urine albumin-to-creatinine ratio (UACR) $\geq 30 \mathrm{mg} / \mathrm{g}$ [ $\geq 3 \mathrm{mg} / \mathrm{mmol}]$ ), urine sediment abnormalities, renal tubular disorders, and pathological and structural abnormalities $[1,2]$. CKD is a common condition and major risk factor for cardiovascular complications, end-stage kidney disease (ESKD), and premature death $[1,2]$. According to 2021 data, the estimated prevalence of CKD in the USA is 15\% (37 million adults), and CKD is more common in older (aged 65 years or more) versus younger individuals and in non-Hispanic Black individuals versus non-Hispanic White individuals or Asians [3]. CKD is frequently underdiagnosed and is associated with low patient and physician awareness. In the ADD-CKD study of adults with type 2 diabetes (T2D), only $22 \%$ of patients with stage 3-5 CKD were identified as having CKD by their primary care physician [4]. This proportion increased as the CKD stage worsened, from $18 \%$ for stage 3, to $53 \%$ for stage 4 , and to $59 \%$ for stage $5 \mathrm{CKD}$ [4]. Additionally underappreciated is the importance of both components of CKD (i.e., eGFR $<60 \mathrm{~mL} / \mathrm{min} /$ $1.73 \mathrm{~m}^{2}$ and $\mathrm{UACR} \geq 30 \mathrm{mg} / \mathrm{g}$ ) as separate independent risk factors for CKD progression, the development of ESKD and cardiovascular disease (CVD), and mortality [5, 6].

There is an unmet need for additional effective treatments for CKD that slow disease progression, prevent development of ESKD and CVD including heart failure (HF), and prolong survival of patients with CKD. In the last 20 years, angiotensin-converting enzyme (ACE) inhibitors and angiotensin receptor blockers (ARBs) have been the only classes of agents recommended for patients with CKD and hypertension, with or without T2D [2]. In the RENAAL (losartan) [7] and IDNT (irbesartan) [8] studies in patients with T2D and nephropathy, 
both ARBs reduced the risk of the composite renal endpoint (i.e., doubling of serum creatinine, ESKD, or all-cause mortality) by $16 \%$ and $20 \%$, respectively, compared with placebo. Similarly, ACE inhibitors have also been associated with reduced risk of CKD progression compared with placebo, both in patients with and without T2D [9-12]. Subsequent studies on ACE inhibitor plus ARB combinations or renin inhibitors have not shown additional benefits with regard to CKD progression or were prematurely terminated because of serious adverse effects (acute kidney injury, renal dysfunction, stroke and/or hyperkalemia) [13-15].

More recently, large, randomized, placebocontrolled studies of sodium-glucose cotransporter 2 (SGLT2) inhibitors in patients with T2D investigated the cardiovascular safety of this class of glucose-lowering therapies. In addition to significantly reducing the risk of cardiovascular events, SGLT2 inhibitors reduced the risk of clinically relevant renal outcomes (sustained loss of kidney function, eGFR decline, progression to or worsening of albuminuria, new ESKD, death from renal causes, and/or a renal composite outcome) compared with placebo, indicating that SGLT2 inhibitors are associated with significantly lower risk of worsening of kidney function [16-19]. However, these studies were not designed to evaluate treatment benefits in patients with CKD, with only $7-26 \%$ of participants having an eGFR of less than $60 \mathrm{~mL} / \mathrm{min} /$ $1.73 \mathrm{~m}^{2}$ [16-19]. Subsequently, designated kidney outcome trials showed a marked reduction in the risk of CKD progression with SGLT2 inhibitors in patients with diabetic kidney disease (DKD) with canagliflozin in CREDENCE [20] and DKD as well as nondiabetic CKD with dapagliflozin in DAPA-CKD [21]. Additionally, the FIDELIO-DKD trial evaluating the long-term effects of the mineralocorticoid antagonist (MRA) finerenone on kidney and cardiovascular outcomes reported a reduced risk of CKD progression in patients with DKD [22].

This review discusses the mechanisms underlying CKD progression and its associated risk factors and provides an overview of potential therapeutic approaches for slowing or preventing CKD progression, with a focus on the evidence supporting the therapeutic use of
SGLT2 inhibitors in patients with CKD, with or without T2D.

\section{METHODS}

A literature search using the PubMed database was conducted to identify English-language articles published between June 2016 and September 2021. The search used free-text terms combined with Boolean operators in PubMed and was limited to the following literature: progression of CKD and its pathogenesis and treatment ("chronic kidney disease" OR "progression of kidney disease" OR "end-stage kidney disease" OR "pathophysiology" OR "diabetic kidney disease" OR "renoprotection") and SGLT2 inhibitors "SGLT2 inhibitor" OR "SGLT-2 inhibitor" OR "sodium-glucose cotransporter 2 inhibitor" OR "canagliflozin" OR "dapagliflozin" OR "empagliflozin" OR "ertugliflozin" OR "sotagliflozin"). The literature search results for SGLT2 inhibitors pertaining to clinical trials and metaanalyses were reviewed; the studies reporting renal and/or cardiovascular outcomes for SGLT2 inhibitors or that were designated kidney outcome trials or had enrolled patients with CKD were preferentially included. This literature assessment was also supplemented with a search of relevant articles about kidney outcome trials pertaining to glucagon-like peptide 1 receptor agonists (GLP1-RAs) and finerenone.

This article is based on previously conducted studies and does not contain any new studies with human participants or animals performed by the author.

\section{PROGRESSION OF CKD}

The mechanisms underlying CKD progression are multifactorial and may be categorized into three major pathways: hemodynamic, metabolic, and inflammatory or fibrotic (Fig. 1) [23]. Glomerular hyperfiltration leads to glomerular hypertension, glomerular cell and mesangial proliferation, macrophage infiltration, accumulation of extracellular matrix, and podocyte injury [24]. In addition, the initial loss of nephrons invokes a compensatory hyperfiltration 


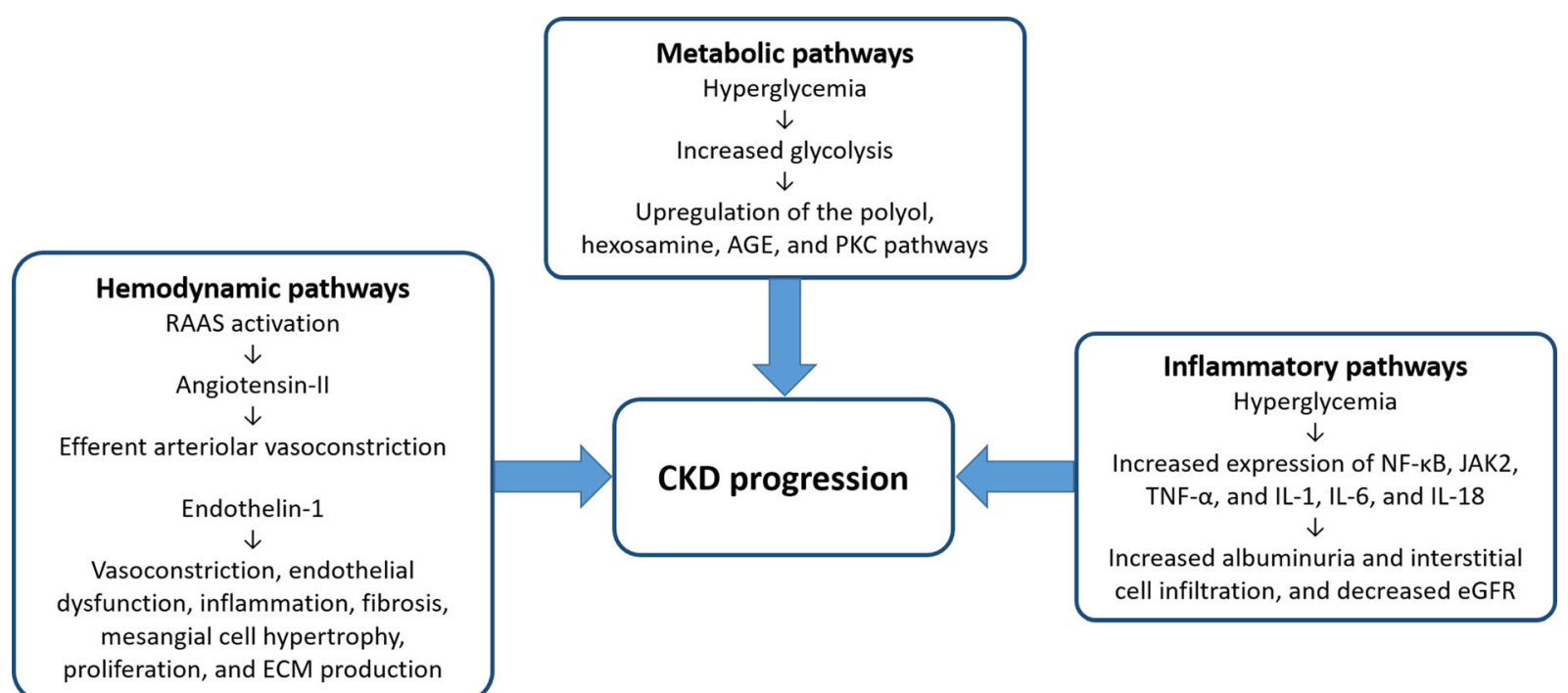

Fig. 1 Major pathways underlying the progression of CKD [23]. $A G E$ advanced glycation end product, $C K D$ chronic kidney disease, $E C M$ extracellular matrix, $e G F R$ estimated glomerular filtration rate, $I L$ interleukin, JAK2

in the residual nephrons. All these factors contribute to glomerulosclerosis, tubulointerstitial fibrosis, and further decline of the eGFR [24].

The 2021 Kidney Disease: Improving Global Outcomes (KDIGO) guidelines recommend monitoring for CKD progression by assessing both eGFR (using serum creatinine levels) and albuminuria at least annually, or more often in those at high risk for progression or for whom assessment will affect treatment decisions [1]. CKD progression is defined as a decrease in GFR category (e.g., from stage 3 a $[45-59 \mathrm{~mL} / \mathrm{min} /$ $\left.1.73 \mathrm{~m}^{2}\right]$ to stage $3 \mathrm{~b}\left[30-44 \mathrm{~mL} / \mathrm{min} / 1.73 \mathrm{~m}^{2}\right]$ ) or a decline in eGFR defined as a decrease in GFR category combined with at least a $25 \%$ reduction in eGFR from baseline. A sustained decline in eGFR of greater than $5 \mathrm{~mL} / \mathrm{min} /$ $1.73 \mathrm{~m}^{2} /$ year is defined as rapid CKD progression [1]. The presence of albuminuria is associated with a faster rate of decline in kidney function [1]. The degree of CKD progression depends on multiple factors: albuminuria, primary cause of CKD, eGFR level, age, sex, race/ ethnicity, obesity, smoking status, presence and control of hypertension, hyperglycemia, dyslipidemia, underlying CVD, and exposure to nephrotoxic medications (e.g., nonsteroidal
Janus kinase 2, $N K-\kappa B$ nuclear factor- $\kappa \mathrm{B}, P K C$ protein kinase C, RAAS renin-angiotensin-aldosterone system, $T N F-\alpha$ tumor necrosis factor- $\alpha$

anti-inflammatory drugs) [1]. Among patients with CKD, the annual decline in eGFR typically ranges from 1 to $5 \mathrm{~mL} / \mathrm{min} / 1.73 \mathrm{~m}^{2} /$ year [1]. In contrast, normal aging is associated with a much slower decline in eGFR of approximately $0.8 \mathrm{~mL} / \mathrm{min} / 1.73 \mathrm{~m}^{2} /$ year [25].

CKD rarely exists as a solitary diagnosis, and comorbidities such as T2D, hypertension, obesity, and hyperlipidemia contribute to CKD progression [26], as well as further increasing the risks of coronary artery disease, HF, arrhythmias, and sudden cardiac death [27]. Dysfunction in the kidneys has major implications for the cardiovascular system (and vice versa), which is a condition known as cardiorenal syndrome [28]. Although T2D and hypertension are the most common causes of CKD development and subsequent progression, other risk factors should also be considered, including race, older age, low birth weight, obesity, smoking status, prior acute kidney injury (AKI), immunoglobulin A nephropathy, systemic lupus erythematosus, and a family history of CKD [29-31]. 


\section{Diabetes and CKD}

The prevalence of CKD as a complication of T2D, also called DKD, is approximately $40 \%$ in the USA [32-34]. One of the key initial mechanisms underlying CKD development in diabetes is hyperglycemia-induced glomerular hyperfiltration [35]. Diabetes induces kidney hypertrophy, mainly through proximal tubular growth and upregulation of SGLT2 transporters, which leads to increased proximal reabsorption of glucose and sodium by SGLT2 transporters. This results in reduced delivery of sodium chloride to the macula densa in the distal tubule (at the loop of Henle) causing afferent (preglomerular) vasodilation and glomerular hyperfiltration via tubule-glomerular feedback. Glomerular hyperfiltration and hypertension cause physical stress (barotrauma) in the glomerulus (leading to increased oxygen demands) and in the tubular cells, which leads to hypertrophy with a proinflammatory and profibrotic phenotype and subsequently tubulointerstitial damage [35]. Upregulation of the systemic and intrinsic renal renin-angiotensin-aldosterone system (RAAS), including proximal tubular angiotensinogen generation and juxtaglomerular renin release, leads to increased angiotensin II and aldosterone levels [36]. Angiotensin II further stimulates proximal tubular reabsorption of sodium and induces efferent arteriolar vasoconstriction, augmenting glomerular hypertension [37]. The pathologic findings of DKD include an initial basement membrane thickening, followed by glomerular mesangial expansion, loss of podocytes, tubular hypertrophy, and glomerular sclerosis, ultimately resulting in tubulointerstitial inflammation and fibrosis [38, 39]. Over the last 20 years, there has been a phenotype change in the clinical manifestations of DKD, with a decreased prevalence of albuminuria, which was surmised to be due to higher usage of diabetes therapies and RAAS inhibitors (ACE inhibitors, ARBs), and an increased prevalence of reduced eGFR in patients with diabetes for which the cause was unknown [34].

\section{Hypertension and CKD}

Hypertension is the second leading cause of ESKD and has a higher prevalence in non-Hispanic Black individuals than in individuals of Hispanic and other non-Hispanic race origin groups [40]. Hypertension is both a cause and consequence of $\mathrm{CKD}$, and depending on the CKD stage, its prevalence ranges between $60 \%$ and 90\% among patients with CKD [41].

Traditionally, kidney damage caused by essential hypertension has two distinct phenotypes [42]. "Benign" nephrosclerosis is caused by nonspecific hyaline arteriolosclerosis that slowly progresses to focal ischemic glomerulosclerosis without overt albuminuria, whereas "malignant" nephrosclerosis occurs in individuals with severe uncontrolled hypertension and is characterized by prevalent fibrinoid necrosis, thrombosis, and ischemic glomerulosclerosis, as well as a more rapid course of CKD progression to ESKD if untreated. However, malignant nephrosclerosis is less frequently observed because of the widespread availability of antihypertensive therapy [42].

Hypertension causes new onset or progression of CKD by several mechanisms. These include direct barotrauma to the glomerulus from uncontrolled hypertension (despite renal autoregulation of systemic blood pressure [BP]) and systemic and renal activation of the RAAS and angiotensin II, causing ischemic glomerulosclerosis and efferent vasoconstriction with glomerular hypertension and hyperfiltration [43]. In CKD, development or acceleration of hypertension is associated with salt retention with volume overload, activation of the sympathetic nervous system, endothelial dysfunction, and oxidative stress [41].

\section{CVD and CKD}

In individuals aged over 65 years, the prevalence of CVD is approximately twice as high among patients with versus without CKD (65\% vs. $32 \%$ ) [44]. Approximately $24 \%$ of US Medicare patients with CKD have comorbid atrial fibrillation (AF), and $51 \%$ of those with CKD and $\mathrm{AF}$ also have a diagnosis of HF [44]. In the 
Kaiser Permanente Renal Registry study of $1,120,295$ individuals for whom at least one serum creatinine measurement was available, reduced eGFR and albuminuria were found to be strong independent risk factors for hospitalization, all-cause mortality, and cardiovascular events (defined as hospitalization for coronary disease, HF, stroke, or peripheral artery disease) [45]. An estimated 50\% of patients with stage 4-5 CKD have CVD, and approximately $40-50 \%$ of all deaths are due to cardiovascular mortality in patients with advanced CKD or ESKD, compared with $26 \%$ in those without CKD [27]. Furthermore, patients with CKD have a higher risk of CVD development than progression to ESKD [27].

Risk factors for CVD in patients with CKD can be classified as "traditional" and "nontraditional" (Table 1) [29, 46, 47]. Traditional cardiovascular risk factors do not have the same predictive value in CKD as in the general population [47].

As mentioned previously, these cardiovascular risk factors may also increase the risk for CKD progression. For example, obesity (body mass index $[\mathrm{BMI}] \geq 30 \mathrm{~kg} / \mathrm{m}^{2}$ ) has been associated with an $83 \%$ increased risk of incident CKD [48], and the risk of ESKD may be increased

Table 1 Traditional and nontraditional risk factors for CVD in patients with CKD $[46,47]$

\begin{tabular}{ll}
\hline Traditional risk factors & Nontraditional risk factors \\
\hline Age & Endothelial dysfunction \\
Male sex & Anemia \\
Left ventricular & Hyperuricemia \\
hypertrophy & \\
Smoking & Inflammation \\
Dyslipidemia & Oxidative stress \\
Hypertension & Vascular calcification \\
Diabetes mellitus & Mineral bone metabolism \\
Physical inactivity & Abnormal lipid \\
& modifications \\
\hline
\end{tabular}

$C K D$ chronic kidney disease, $C V D$ cardiovascular disease approximately sevenfold in individuals with class III obesity (BMI $\left.\geq 40 \mathrm{~kg} / \mathrm{m}^{2}\right)$ [49]. The potential mechanisms underlying this increased risk for CKD and ESKD include obesity-mediated hypertension, inflammation, glomerular hyperfiltration, activation of the RAAS (including elevated aldosterone levels), insulin resistance, dysregulation of adipocytokines, and consequences of extra- and intrarenal ectopic fat depositions described in fatty kidney disease $[50,51]$.

\section{THERAPEUTIC APPROACHES TO SLOWING CKD PROGRESSION}

To slow the progression of CKD, key drivers and factors responsible for disease progression must be addressed, including management of hyperglycemia in T2D, hypertension, albuminuria, obesity, and other risk factors.

\section{Hyperglycemia Control in T2D}

Adequate control of glycated hemoglobin (HbA1c) is important in patients with T2D to reduce the risk of microvascular complications, including nephropathy, retinopathy, and neuropathy, as well as new-onset albuminuria and its progression [52]. Although DKD develops because of long-standing uncontrolled hyperglycemia, intensive lowering of $\mathrm{HbA1c}$ to $\leq 6.5 \%$ was shown to only modestly slow CKD progression in patients with T2D. In the ADVANCE trial in more than 11,000 patients with $\mathrm{T} 2 \mathrm{D}$, intensive glucose control (target HbA1c $\leq 6.5 \%)$ significantly reduced the 5-year risk of ESKD by $65 \%$ (7 vs. 20 events) and macroalbuminuria by $30 \% \quad(162$ vs. 231 patients) versus standard glucose control [53]. In ADVANCE-ON, a post-trial follow-up of ADVANCE in approximately 8500 patients (median total follow-up 9.9 years), despite an increase in HbA1c levels to $7.3 \%$ in the prior intensive-control group, there was still significant reduction in risk of ESKD by $46 \%$ ( 29 vs. 53 events) [54]. However, importantly these benefits were mostly seen in patients with baseline 
Table 2 Clinical scenarios where the risks of an SBP target of $<120 \mathrm{mmHg}$ may outweigh the benefits, according to the 2021 Kidney Disease: Improving Global Outcomes guidelines [2]

\begin{tabular}{|c|c|}
\hline Clinical scenario & Reason for uncertainty \\
\hline $\begin{array}{l}\text { Patients with stage } 4 \text { or } 5 \\
\text { CKD }\end{array}$ & $\begin{array}{l}\text { There is less certainty around the benefit of a lower BP target and the potential risks in } \\
\text { patients with lower vs. higher eGFR }\end{array}$ \\
\hline Diabetes & $\begin{array}{l}\text { Benefits of intensive BP lowering are less certain in patients with CKD who have diabetes } \\
\text { vs. those who do not have diabetes }\end{array}$ \\
\hline $\begin{array}{l}\text { Patients with SBP of } \\
120-129 \mathrm{mmHg}\end{array}$ & $\begin{array}{l}\text { These patients may be at higher cardiovascular risk than those with SBP }<120 \mathrm{mmHg} \\
\text { and may hypothetically benefit, but RCTs in CKD have not included patients with SBP } \\
\text { of } 120-129 \mathrm{mmHg}\end{array}$ \\
\hline $\begin{array}{l}\text { Patients with baseline } \\
\text { DBP }<50 \mathrm{mmHg}\end{array}$ & $\begin{array}{l}\text { Intensive } \mathrm{BP} \text { lowering may increase the risk of myocardial infarction, particularly in } \\
\text { patients with CAD }\end{array}$ \\
\hline Etiology of CKD & $\begin{array}{l}\text { In autosomal dominant polycystic kidney disease, kidney benefits may be greater with an } \\
\text { SBP of } 95-110 \mathrm{mmHg} \text { vs. } 120-130 \mathrm{mmHg}\end{array}$ \\
\hline Albuminuria & $\begin{array}{l}\text { Albuminuria is no longer considered an effect modifier of BP target with an SBP target of } \\
<120 \mathrm{mmHg}\end{array}$ \\
\hline Older age & In patients aged $>90$ years, the risk-to-benefit ratio is less certain because of limited data \\
\hline Younger age & $\begin{array}{l}\text { In patients aged }<50 \text { years who may have absolute risks of CVD and all-cause mortality, } \\
\text { the risk-to-benefit ratio is less certain }\end{array}$ \\
\hline Frailty & $\begin{array}{l}\text { For patients who are very frail or living in a nursing home, frailty does not appear to } \\
\text { modify the benefits of intensive BP lowering }\end{array}$ \\
\hline "White-coat" hypertension & $\begin{array}{l}\text { If } \mathrm{BP} \text { measured in the office is higher than ambulatory } \mathrm{BP} \text { or } \mathrm{BP} \text { measured at home, } \\
\text { additional BP lowering to achieve in-office SBP }<120 \mathrm{mmHg} \text { may increase risks, with } \\
\text { less certain benefits }\end{array}$ \\
\hline Severe hypertension & $\begin{array}{l}\text { Patients with SBP } \geq 180 \mathrm{mmHg} \text { on } \leq 1 \text { antihypertensive drug or } \geq 150 \mathrm{mmHg} \text { on }>4 \\
\text { antihypertensive drugs were excluded from SPRINT }\end{array}$ \\
\hline
\end{tabular}

$B P$ blood pressure, $C A D$ coronary artery disease, $C K D$ chronic kidney disease, $C V D$ cardiovascular disease, $D B P$ diastolic blood pressure, $e G F R$ estimated glomerular filtration rate, $R C T$ randomized clinical trial, $S B P$ systolic blood pressure

eGFR $\geq 60 \mathrm{~mL} / \mathrm{min} / 1.73 \mathrm{~m}^{2}$ and systolic $\mathrm{BP}$ (SBP) $<140 \mathrm{mmHg}$ [54].

\section{Hypertension Management}

Uncontrolled hypertension increases the risk for cardiovascular events and is a major risk factor for CKD, but the ideal target $\mathrm{BP}$ in patients with CKD remains unclear. In the SPRINT trial of 9361 patients with SBP $\geq 130 \mathrm{mmHg}$ and increased cardiovascular risk (without T2D), intensive treatment (SBP target $<120 \mathrm{mmHg}$ ) was associated with $25 \%$ reduction in the risk of cardiovascular events (i.e., myocardial infarction [MI], acute coronary syndrome, stroke, HF, or death from cardiovascular causes) compared with standard treatment (SBP target $<140 \mathrm{mmHg}$ ) [55]. However, there was no significant difference in CKD progression (i.e., $\geq 50 \%$ eGFR reduction, ESKD, or kidney transplant) between intensive and standard treatment [55].

The ACCORD BP trial in 4733 patients with T2D and high cardiovascular risk reported an 
SBP target of $<120 \mathrm{mmHg}$ was not superior to that of $<140 \mathrm{mmHg}$ for reducing risk of cardiovascular events (i.e., nonfatal MI, nonfatal stroke, or cardiovascular death) [56], and was associated with a greater decline in eGFR over 2 years among a subgroup of participants with CKD $(n=529)$ [57].

The American College of Cardiology/American Heart Association 2017 guideline for hypertension management recommends a BP goal of $<130 / 80 \mathrm{mmHg}$ in patients with CKD (with or without diabetes) [58]. However, the KDIGO 2021 guidelines state a lower SBP goal $(<120 \mathrm{mmHg})$ in patients with $\mathrm{CKD}$ (2C recommendation) but list many areas of uncertainty, including advanced CKD, T2D, an SBP of 120-129 mmHg, very low diastolic $\mathrm{BP}$ (e.g., $<50 \mathrm{mmHg}$ ), albuminuria, "white-coat" hypertension, or severe hypertension (e.g., SBP $\geq 180 \mathrm{mmHg}$ on at most one antihypertensive drug) and age $<50$ years, age $>90$ years, or frailty (Table 2) [2]. Considering all the data, a BP goal of $\leq 130 / 80 \mathrm{mmHg}$ appears most appropriate.

The KDIGO 2021 guidelines recommend lifestyle interventions for lowering BP in patients with $\mathrm{CKD}$, including restricting sodium intake to $<2 \mathrm{~g}(<90 \mathrm{mmol}$ sodium or $<5 \mathrm{~g}$ sodium chloride) per day and undertaking moderate physical activity for up to $150 \mathrm{~min} /$ week, depending on cardiovascular status and physical fitness levels [2]. When medication is indicated, initiating hypertensive treatment with an ACE inhibitor or $\mathrm{ARB}$ titrated to a maximum tolerated dose is recommended in patients with CKD and albuminuria (with or without diabetes), followed by addition of a diuretic and/or calcium channel blocker [2]. Non-dihydropyridine calcium channel blockers, such as diltiazem and verapamil, may be beneficial in patients with albuminuria [59]. Although not classified as antihypertensive drugs, SGLT2 inhibitors (discussed in detail later) have additionally been associated with SBP reductions of approximately $4 \mathrm{mmHg}$ in $\mathrm{T} 2 \mathrm{D}$ [60].

\section{Reducing Albuminuria}

Albuminuria is a sign of system-wide endothelial dysfunction $[61,62]$ and is independent of
eGFR and other cardiovascular risk factors associated with increased cardiovascular and allcause mortality [63]. In the last 15 years, studies have focused on albuminuria reduction to slow CKD progression and prevent CVD. In a metaanalysis conducted by the REASSURE Consortium, each $30 \%$ reduction in albuminuria reduced the risk of ESKD by $24 \%$, irrespective of the drug class used [64]. In 2020, a National Kidney Foundation, US Food and Drug Administration, and European Medicines Agency workshop accepted a geometric mean albuminuria reduction of $30 \%$ within 6 months (or an eGFR slope reduction of $0.5-1.0 \mathrm{~mL} / \mathrm{min} /$ $1.73 \mathrm{~m}^{2} /$ year over $2-3$ years) as a surrogate renal endpoint marker of treatment effect for CKD progression in clinical trials [6]. A post hoc analysis of the RENAAL study found that each $50 \%$ reduction in albuminuria with losartan reduced the risk of $\mathrm{HF}$ by $27 \%$ and the risk of cardiovascular events by $18 \%$ [65].

Currently, five drug classes are available that have shown at least a $30 \%$ reduction in albuminuria or UACR, as well as direct evidence of the slowing of CKD progression or a reduction in eGFR decline in dedicated trials of patients with T2D and CKD, namely ACE inhibitors [66], ARBs [8], SGLT2 inhibitors (discussed in detail later) [16, 21, 67], GLP1-RAs [68], and the MRA finerenone [22]. Dipeptidyl peptidase 4 (DPP4) inhibitors are another class of glucose-lowering therapy that reduce albuminuria $(<30 \%)$ in patients with T2D $[69,70]$. A systematic review and meta-analysis, which included 23 randomized clinical trials $(N=41,359$ patients), reported that DPP4 inhibitors demonstrated a renoprotective effect due to a lower risk of albuminuria development or progression [69]. However, these agents have not been proven to reduce the risk of CKD progression or ESKD $[69,70]$.

\section{Management of Obesity}

In patients with $\mathrm{T} 2 \mathrm{D}$, the obesity rate is double that of the US general population ( $89 \%$ vs. $42 \%$ ) $[71,72]$, and obesity at 20 years confers a 3.6fold increased risk of CKD [73]. Weight reduction has been shown to reduce albuminuria and 
provide renal benefits, as well as cardiovascular benefits, in overweight or obese patients with CKD $[74,75]$. In systematic reviews of studies in patients with CKD or in overweight or obese patients with $\mathrm{CKD}$, dietary restriction-induced weight loss decreased overt proteinuria or microalbuminuria, and bariatric surgery normalized glomerular hyperfiltration [74, 75]. However, no studies have shown weight loss to significantly slow the progression of CKD $[74,75]$.

\section{Management of Other Risk Factors}

KDIGO recommendations call for at least $150 \mathrm{~min} /$ week of exercise, discontinuation of smoking, and avoidance of nephrotoxic drugs (e.g., nonsteroidal anti-inflammatory drugs) in patients with CKD [1]. Dietary protein restriction to $<1.3 \mathrm{~g} / \mathrm{kg}$ for eGFR $<60 \mathrm{~mL} / \mathrm{min} /$ $1.73 \mathrm{~m}^{2}$ and $<0.8 \mathrm{~g} / \mathrm{kg}$ for eGFR $<30 \mathrm{~mL} / \mathrm{min} /$ $1.73 \mathrm{~m}^{2}[1]$ and an increase in consumption of fresh fruits and vegetables are suggested, although the benefits of these recommendations have not been proven in clinical trials. Treatment of dyslipidemia by lowering lowdensity lipoprotein cholesterol levels with statin-based regimens reduces the risk of cardiovascular events in patients with CKD but has not been shown to slow CKD progression [76]. Similarly, treatment of anemia with erythropoiesis-stimulating agents (erythropoietin-alfa, erythropoietin-beta, or darbepoetin) in patients with CKD was not associated with significant slowing of CKD progression to ESKD [77]. In contrast, correction of CKD-associated metabolic acidosis with sodium bicarbonate therapy has been shown to slow progression of CKD [78].

\section{NEW THERAPEUTIC AGENTS FOR SLOWING CKD PROGRESSION}

In addition to treatment strategies that address hyperglycemia, hypertension, albuminuria, obesity, and other comorbidities, there is emerging evidence of the benefits of other therapeutic agents for slowing CKD progression and improving outcomes in patients with CKD, including SGLT2 inhibitors [20, 21], the MRA finerenone [22], and GLP1-RAs [68].

\section{Effect of SGLT2 Inhibitors on CKD Progression}

Several large cardiovascular outcomes trials in patients with T2D and predominantly preserved kidney function showed a reduced risk of CKD progression with the SGLT2 inhibitors empagliflozin (in EMPA-REG OUTCOME) [17], canagliflozin (in CANVAS) [16], and dapagliflozin (in DECLARE-TIMI 58) [18]. More recently, a marked reduction in the risk of CKD progression with SGLT2 inhibitors was confirmed with canagliflozin in patients with DKD (in CREDENCE) [20] and with dapagliflozin in patients with DKD or nondiabetic CKD (in DAPA-CKD and DECLARE-TIMI 58) [19, 21, 79].

In the CREDENCE study in 4401 patients with T2D and albuminuric CKD (eGFR 30 to $<90 \mathrm{~mL} / \mathrm{min} / 1.73 \mathrm{~m}^{2}$; $\quad$ UACR $\geq 300 \quad$ to $5000 \mathrm{mg} / \mathrm{g}$ ), canagliflozin $100 \mathrm{mg}$ once daily significantly reduced the risk of the primary composite endpoint (i.e., ESKD, doubling of serum creatinine, or renal or cardiovascular death) by $30 \%$ compared with placebo $(P<0.001)$ [20]. In addition, there was a significant reduction in the risk of ESKD by $32 \%$ $(P=0.002)$ and of the composite endpoint of ESKD, doubling of serum creatinine, or renal death by $34 \%(P<0.001)$ [20].

In the DAPA-CKD study in 4304 patients with CKD, either with $(67.5 \%)$ or without (32.5\%) T2D, dapagliflozin $10 \mathrm{mg}$ once daily significantly reduced the risk of the primary composite endpoint (i.e., sustained $50 \%$ or more decline in eGFR, ESKD, or renal or cardiovascular death) by $39 \%$ compared with placebo $(P<0.001)$ [21]. Further, dapagliflozin significantly reduced the risk of the secondary composite endpoint of $50 \%$ or more decline in eGFR, ESKD, or renal death by $44 \%(P<0.001)$ and of all-cause mortality by $31 \%(P=0.004)$ [21]. Importantly, DAPA-CKD was the first trial to show reduction in risk of all-cause mortality in patients with CKD, and also the first trial of an SGLT2 inhibitor to demonstrate 
improvement in kidney disease outcomes, being equally effective in patients with T2D and CKD and in those with nondiabetic CKD [80].

In the DECLARE-TIMI 58 study of patients with DKD or nondiabetic CKD, the effects of dapagliflozin compared with placebo on renal outcomes were evaluated on the basis of a composite cardiorenal (sustained decline of at least $40 \%$ in eGFR $>60 \mathrm{~mL} / \mathrm{min} / 1.73 \mathrm{~m}^{2}$, ESKD, or death from renal or cardiovascular causes) and composite renal-specific (excluding cardiovascular death) outcomes $[18,19,79]$. The dapagliflozin group had a significantly reduced frequency of the composite cardiorenal outcome [HR 0.76 (95\% CI 0.67-0.87); $P<0.0001$ ] and the renal-specific outcome [HR 0.53 (95\% CI 0.43-0.66); $P<0.0001]$ compared with the placebo group [19]. There was a significantly lower risk of a sustained eGFR decline by at least $40 \%$ to eGFR $<60 \mathrm{~mL} / \mathrm{min} / 1.73 \mathrm{~m}^{2}$ [HR 0.54 (95\% CI 0.43-0.67); $P<0.0001]$ for dapagliflozin versus placebo [19] and fewer patients had ESKD in the dapagliflozin group than the placebo group [HF 0.41 (95\% CI $0.20-0.82)$; $P=0.012$ ] [19]. Long-term (baseline to 4 years) changes in UACR were significantly improved with dapaglifozin versus placebo [HR 1.45 (95\% CI 1.35-1.56), $P<0.0001]$ irrespective of UACR or eGFR at baseline [79]. In the dapagliflozin group compared with the placebo group, there were significant reductions in the composite cardiorenal outcome across subgroups of $\mathrm{UACR} \geq 30 \mathrm{mg} / \mathrm{g}(P<0.0125)$ as well as in the renal-specific outcome across all UACR categories $(P<0.05)[79]$.

\section{Treatment of CKD with SGLT2 Inhibitors in Clinical Practice}

On the basis of the findings from CREDENCE [20], DECLARE-TIMI 58 [18, 19], and DAPACKD [21], the US Food and Drug Administration expanded approval of canagliflozin to include patients with T2D and diabetic nephropathy, with albuminuria $>300 \mathrm{mg} /$ day and an eGFR $\geq 30 \mathrm{~mL} / \mathrm{min} / 1.73 \mathrm{~m}^{2}$ [81], and approval of dapagliflozin to include patients with CKD with an $\mathrm{eGFR} \geq 25 \mathrm{~mL} / \mathrm{min} / 1.73 \mathrm{~m}^{2}$ at risk of CKD progression [18, 19, 82]. Therefore, it is important that all healthcare providers, including primary care physicians and nephrologists, are aware of the therapeutic value that SGLT2 inhibitors add to disease management in patients with CKD.

Although canagliflozin is indicated to reduce the risk of CKD progression (i.e., ESKD or doubling of serum creatinine), cardiovascular death, and hospitalization for HF (HHF) only in patients with T2D and albuminuria $\geq 300 \mathrm{mg} / \mathrm{g}$ [81], dapagliflozin is indicated to reduce the risk of CKD progression (i.e., ESKD or sustained eGFR decline) and HHF irrespective of albuminuria in all patients with CKD (with or without T2D), as well as to reduce the risk of cardiovascular death and HHF in patients with HF with reduced ejection fraction [82].

SGLT2 inhibitors are associated with a reduction in glomerular hyperfiltration, likely caused by an increase in preglomerular vasoconstriction and a decrease in postglomerular vascular resistance [83]; therefore, an initial decrease in eGFR of approximately $4-5 \mathrm{~mL} /$ $\min / 1.73 \mathrm{~m}^{2}$ is expected within the first 2-3 weeks of SGLT2 inhibitor therapy. A study in patients with type 1 diabetes suggested that this decrease in eGFR is secondary to adenosineinduced preglomerular vasoconstriction [84], whereas a more recent study in patients with T2D indicated that a decline in eGFR may additionally be caused by postglomerular vasodilation [83]. In the latter study, the decrease in eGFR corresponded to a reduction in intraglomerular pressure of $1.4 \mathrm{mmHg}$ in euglycemia and $1.9 \mathrm{mmHg}$ in hyperglycemia [83]. This reduction in intraglomerular pressure is similar to that observed with ACE inhibitor or ARB therapy [85]. In CREDENCE and DAPACKD, canagliflozin or dapagliflozin was associated with an initial mean eGFR decline of $3.7 \mathrm{~mL} / \mathrm{min} / 1.73 \mathrm{~m}^{2}$ within 3 weeks or $4.0 \mathrm{~mL} /$ $\mathrm{min} / 1.73 \mathrm{~m}^{2}$ within 2 weeks, respectively, after which the decline stabilized and the annual change in eGFR was less with the SGLT2 inhibitor than with placebo $[20,21]$. This initial dip in eGFR was reported to exceed $10 \%$ in one out of four patients, but is not expected to lead to discontinuation of SGLT2 inhibitor therapy, unless eGFR decreases by $30 \%$ or more $[85,86]$. An eGFR dip of greater than $10 \%$ was typically 
seen in patients with more depleted volume status receiving concomitant diuretics and with more advanced CKD stages, but importantly this did not affect kidney or cardiovascular outcomes [86].

An important consideration for SGLT2 inhibitors in patients with CKD is the potential for SGLT2 inhibitor-related adverse events. There is an increased risk of non-serious adverse events with this class, including urinary tract infections and genital mycotic infections, compared with placebo [81, 82]; however, the risk of serious adverse events is low.

The risk of diabetic ketoacidosis (DKA), frequently presenting with euglycemia, may be increased with SGLT2 inhibitors, particularly in patients with restricted carbohydrate intake (e.g., ketogenic diet) [87], insulin deficiency, or history of alcohol abuse [81, 82]. In CREDENCE, the rate of DKA was higher with canagliflozin than with placebo (2.2 vs. 0.2 per 1000 patientyears) [20], but in DAPA-CKD, there were no cases of DKA reported with dapagliflozin (vs. two with placebo) [21].

Although there have been postmarketing reports of AKI related to volume depletion with canagliflozin and dapagliflozin in patients with T2D [81, 82], the risk of AKI with SGLT2 inhibitors appears to be low. In DAPA-CKD, the cumulative incidence of AKI (32 months of follow-up) was lower with dapagliflozin than with placebo [2.9\% vs. $4.2 \%$; HR 0.68 (95\% CI 0.49-0.94)] [67, 88]. In CREDENCE, the incidence of AKI was similar in the canagliflozin and placebo groups [16.9 vs. 20.0 events/ 1000 patient-years; HR 0.85 (95\% CI 0.64-1.13)] [20]. In light of these data, the US prescribing information for canagliflozin and dapagliflozin no longer includes a warning regarding the risk of AKI; however, a new warning is included to monitor for signs and symptoms of volume depletion for the class $[81,82,89]$. Furthermore, a recent meta-analysis of 18 trials $(N=156,690)$ showed that the risk of AKI with SGLT2 inhibitors was $24 \%$ lower versus placebo, $32 \%$ lower versus DPP4 inhibitors, and $21 \%$ lower versus GLP1-RAs [90].

An initial increase in diuresis at the start of SGLT2 inhibitor therapy should be anticipated to avoid volume depletion and low SBP, especially in patients with eGFR $<60 \mathrm{~mL} /$ $\min / 1.73 \mathrm{~m}^{2}$, older patients, or those on loop diuretics $[81,82]$. SGLT2 inhibitors cause glucosuria-induced osmotic diuresis and natriuresis, which can lead to a total fluid loss of about $1-2 \mathrm{~kg}$ in the first $1-2$ weeks of treatment that subsequently stabilizes [91]. Volume depletion should be corrected before initiation of an SGLT2 inhibitor and a reduction in diuretic therapy considered. This increase in diuresis is not associated with any notable electrolyte disorders [92]. In CREDENCE, the incidence of volume depletion was higher with canagliflozin than with placebo ( 28.4 vs. 23.5 events/1000 patient-years [93]. Similarly, in DAPA-CKD, volume depletion was reported in a higher proportion of patients in the dapagliflozin group than in the placebo group (5.9\% vs. $4.2 \%)$ [21]. Canagliflozin lowered SBP by approximately $3.5 \mathrm{mmHg}$ versus placebo in the CREDENCE study, irrespective of concomitant antihypertensive therapy [94]. The mechanism for this SBP reduction is unclear but appears to be related to vasodilation without an increase in pulse rate [91] and reduction of sympathetic tone [95].

Canagliflozin has been associated with an increased risk of lower limb amputations and fractures in patients with T2D [16, 96]; however, the rates of lower limb amputation and fractures with canagliflozin were similar to those observed with placebo in patients with CKD in CREDENCE [20]. Similarly, dapagliflozin was not associated with an increased risk of amputation or fracture compared with placebo in DAPA-CKD [21]. This suggests that the risks of lower limb amputation and fractures are not increased with canagliflozin or dapagliflozin in patients with CKD.

When used as monotherapy in patients with T2D, the risk of hypoglycemia with SGLT2 inhibitors is low because of their insulin-independent mechanism of action but may be increased when used in combination with insulin or an insulin secretagogue (e.g., sulfonylurea) [81, 82]. Similar rates of hypoglycemia (severity not specified) were observed with canagliflozin and placebo $(10.2 \%$ vs. $10.9 \%$ ) in patients with CKD in CREDENCE [20], and the incidence of major hypoglycemia 
with dapagliflozin was lower than that with placebo $(0.7 \%$ vs. $1.3 \%)$ in DAPA-CKD [21]. There were no reports of major hypoglycemia or DKA with dapagliflozin in patients without T2D in DAPA-CKD [21].

The risk of urinary tract infections and genital mycotic infections is increased with SGLT2 inhibitors because of the increased urinary excretion of glucose, and genital mycotic infection is common in women with a past history [97]. In CREDENCE, the rate of urinary tract infections was similar with canagliflozin versus placebo, but the rate of genital mycotic infections was higher with canagliflozin in both men $(8.4$ vs. 0.9 per 1000 patient-years) and women (12.6 vs. 6.1 per 1000 patient-years) [20]. In DAPA-CKD, serious urinary tract infections were rare, with an incidence of $0.9 \%$ with dapagliflozin versus $0.7 \%$ with placebo, and no serious genital mycotic infections were reported [21].

The benefits of SGLT2 inhibitors in slowing CKD progression versus the risks for treatmentrelated adverse effects necessitate individualization of treatment. When initiating SGLT2 inhibitors for CKD, physicians should assess the benefits of reducing CKD progression, including delay to ESKD and reduction in all-cause mortality with the potential risk of an initial increase in diuresis, DKA, and urinary tract and genital mycotic infections as well as take into consideration underlying comorbidities and concomitant medications.

\section{Other Potential Therapeutic Agents in CKD}

Other drug classes have shown potential for improved outcomes in patients with CKD, including MRAs and GLP1-RAs.

The MRA finerenone was recently approved in patients with CKD and T2D to reduce the risk of CKD progression, cardiovascular death, nonfatal MI, and HHF [98]. This approval was based on the findings of the FIDELIO-DKD study, in which the efficacy and safety of finerenone was evaluated in 5674 patients with T2D and either moderately albuminuric CKD (UACR 30 to $<300 \mathrm{mg} / \mathrm{g}$ and eGFR 25 to $<60 \mathrm{~mL} / \mathrm{min} / 1.73 \mathrm{~m}^{2}$ ) and diabetic retinopathy or severely albuminuric CKD
(UACR $\geq 300$ to $5000 \mathrm{mg} / \mathrm{g}$ and eGFR 25 to $<75 \mathrm{~mL} / \mathrm{min} / 1.73 \mathrm{~m}^{2}$ ) [22]. In FIDELIO-DKD, finerenone was associated with a significantly reduced risk of CKD progression (i.e., kidney failure, sustained at least $40 \%$ decrease in eGFR, or renal death) compared with placebo, as well as a $31 \%$ greater reduction in UACR from baseline after 4 months [22]. However, the risk of hyperkalemia with finerenone increases with decreasing kidney function, particularly in patients with higher potassium levels prior to initiating therapy [98]. In FIDELIO-DKD, the incidence of hyperkalemia was higher with finerenone than with placebo (15.8\% vs. $7.8 \%)$ and more patients discontinued treatment because of hyperkalemia with finerenone versus placebo ( $2.3 \%$ vs. $0.9 \%)$ [22].

In studies of patients with $\mathrm{T} 2 \mathrm{D}$ and predominantly preserved renal function (only $22-23 \%$ had an eGFR $<60 \mathrm{~mL} / \mathrm{min} / 1.73 \mathrm{~m}^{2}$ ), GLP1-RA therapy was associated with significant reductions in UACR or the risk of newonset macroalbuminuria, but there was no significant reduction in the risk of CKD progression or ESKD compared with placebo [99-101]. In comparison, in the AWARD-7 study in patients with T2D and moderate-to-severe CKD (eGFR 15 to $>60 \mathrm{~mL} / \mathrm{min} / 1.73 \mathrm{~m}^{2}$ ), dulaglutide was associated with a significantly smaller decline in eGFR and greater reductions in UACR compared with insulin glargine [68]. The potential benefits of GLP1-RAs in patients with CKD are being further investigated in the FLOW study, a designated renal outcome study of semaglutide in patients with T2D and CKD that is currently in progress (NCT03819153; expected completion August 2024).

\section{CONCLUSIONS}

There is an unmet need for effective treatments to slow CKD progression in patients with CKD (with or without T2D). Until now ACE inhibitors and ARBs have been the only class of agents to be associated with a reduced risk of CKD progression; however, neither has been shown to reduce the risk of all-cause mortality. The multifactorial mechanisms underlying CKD progression must be considered in order to 
effectively manage and slow disease progression. The CREDENCE and DAPA-CKD studies showed a significant reduction in the risk of CKD progression with the SGLT2 inhibitors canagliflozin and dapagliflozin in patients with CKD, with DAPA-CKD being the first trial to report that SGLT2 inhibitors are effective in patients with and without T2D for slowing CKD progression and reducing the risk of all-cause mortality. On the basis of these data, individualized treatment with SGLT2 inhibitors represents an unprecedented therapeutic option and an opportunity to slow the progression of CKD and the development of associated cardiovascular complications in patients with CKD, whether or not they also have T2D.

\section{ACKNOWLEDGEMENTS}

Funding. The development of this manuscript was supported by AstraZeneca. AstraZeneca also funded the journal's Rapid Publication Service fee and Open Access fee.

\section{Medical Writing and Editorial Assistance.}

Medical writing and editorial assistance was provided by Sarah Greig, Ph.D., and Susan M. Kaup, Ph.D., of inScience Communications, Springer Healthcare in accordance with Good Publication Practice (GPP-3), and funded by AstraZeneca.

Authorship. The author meets the International Committee of Medial Journal Editors criteria for authorship for this article, takes responsibility for the integrity of the work as a whole, and has given his approval for this version to be published.

Authorship Contributions. Christian W. Mende contributed to the development of the manuscript, critically reviewed all drafts, and approved the final version of the manuscript for submission.

Disclosures. Christian W. Mende has participated in speaker bureaus or advisory boards for AstraZeneca, Bayer, Boehringer Ingelheim, Lilly, and Janssen.
Compliance with Ethics Guidelines. This article is based on previously conducted studies and does not contain any new studies with human participants or animals performed by the author.

Data Availability. Data sharing is not applicable to this article as no datasets were generated or analyzed during the current study.

Open Access. This article is licensed under a Creative Commons Attribution-NonCommercial 4.0 International License, which permits any non-commercial use, sharing, adaptation, distribution and reproduction in any medium or format, as long as you give appropriate credit to the original author(s) and the source, provide a link to the Creative Commons licence, and indicate if changes were made. The images or other third party material in this article are included in the article's Creative Commons licence, unless indicated otherwise in a credit line to the material. If material is not included in the article's Creative Commons licence and your intended use is not permitted by statutory regulation or exceeds the permitted use, you will need to obtain permission directly from the copyright holder. To view a copy of this licence, visit http://creativecommons.org/licenses/by$\mathrm{nc} / 4.0 /$.

\section{REFERENCES}

1. Kidney Disease: Improving Global Outcomes Work Group. KDIGO. Clinical practice guideline for the evaluation and management of chronic kidney disease. Kidney Int Suppl. 2012;2013(3):1-150.

2. Kidney Disease: Improving Global Outcomes Blood Pressure Work Group. KDIGO. clinical practice guideline for the management of blood pressure in chronic kidney disease. Kidney Int. 2021;2021(99): S1-87.

3. Centers for Disease Control and Prevention. Chronic kidney disease in the United States, 2021. https://www.cdc.gov/kidneydisease/publicationsresources/ckd-national-facts.html. Accessed $17 \mathrm{Aug}$ 2021.

4. Szczech LA, Stewart RC, Su HL, et al. Primary care detection of chronic kidney disease in adults with 
type-2 diabetes: the ADD-CKD Study (awareness, detection and drug therapy in type 2 diabetes and chronic kidney disease). PLoS One. 2014;9:e110535.

5. Blecker S, Matsushita K, Kottgen A, et al. Highnormal albuminuria and risk of heart failure in the community. Am J Kidney Dis. 2011;58:47-55.

6. Levey AS, Gansevoort RT, Coresh J, et al. Change in albuminuria and GFR as end points for clinical trials in early stages of CKD: a scientific workshop sponsored by the National Kidney Foundation in collaboration with the US Food and Drug Administration and European Medicines Agency. Am J Kidney Dis. 2020;75:84-104.

7. Brenner BM, Cooper ME, de Zeeuw D, et al. Effects of losartan on renal and cardiovascular outcomes in patients with type 2 diabetes and nephropathy. N Engl J Med. 2001;345:861-9.

8. Lewis EJ, Hunsicker LG, Clarke WR, et al. Renoprotective effect of the angiotensin-receptor antagonist irbesartan in patients with nephropathy due to type 2 diabetes. N Engl J Med. 2001;345:851-60.

9. Heart Outcomes Prevention Evaluation (HOPE) Study Investigators. Effects of ramipril on cardiovascular and microvascular outcomes in people with diabetes mellitus: results of the HOPE study and MICRO-HOPE substudy. Lancet. 2000;355: 253-9.

10. Jafar TH, Stark PC, Schmid CH, et al. Progression of chronic kidney disease: the role of blood pressure control, proteinuria, and angiotensin-converting enzyme inhibition: a patient-level meta-analysis. Ann Intern Med. 2003;139:244-52.

11. Maschio G, Alberti D, Janin G, et al. Effect of the angiotensin-converting-enzyme inhibitor benazepril on the progression of chronic renal insufficiency. N Engl J Med. 1996;334:939-45.

12. Ruggenenti P, Perna A, Gherardi G, et al. Renoprotective properties of ACE-inhibition in non-diabetic nephropathies with non-nephrotic proteinuria. Lancet. 1999;354:359-64.

13. Fried LF, Emanuele N, Zhang JH, et al. Combined angiotensin inhibition for the treatment of diabetic nephropathy. N Engl J Med. 2013;369:1892-903.

14. ONTARGET Investigators, Yusuf S, Teo KK, et al. Telmisartan, ramipril, or both in patients at high risk for vascular events. N Engl J Med. 2008;358: 1547-59.

15. Parving HH, Brenner BM, McMurray JJ, et al. Cardiorenal end points in a trial of aliskiren for type 2 diabetes. N Engl J Med. 2012;367:2204-13.
16. Perkovic V, de Zeeuw D, Mahaffey KW, et al. Canagliflozin and renal outcomes in type 2 diabetes: results from the CANVAS Program randomised clinical trials. Lancet Diabetes Endocrinol. 2018;6:691-704.

17. Wanner C, Inzucchi SE, Lachin JM, et al. Empagliflozin and progression of kidney disease in type 2 diabetes. N Engl J Med. 2016;375:323-34.

18. Wiviott SD, Raz I, Bonaca MP, et al. Dapagliflozin and cardiovascular outcomes in type 2 diabetes. N Engl J Med. 2019;380:347-57.

19. Mosenzon O, Wiviott SD, Cahn A, et al. Effects of dapagliflozin on development and progression of kidney disease in patients with type 2 diabetes: an analysis from the DECLARE-TIMI 58 randomised trial. Lancet Diabetes Endocrinol. 2019;7:606-17.

20. Perkovic V, Jardine MJ, Neal B, et al. Canagliflozin and renal outcomes in type 2 diabetes and nephropathy. N Engl J Med. 2019;380:2295-306.

21. Heerspink HJ, Stefansson BV, Correa-Rotter R, et al. Dapagliflozin in patients with chronic kidney disease. N Engl J Med. 2020;383:1436-46.

22. Bakris GL, Agarwal R, Anker SD, et al. Effect of finerenone on chronic kidney disease outcomes in type 2 diabetes. N Engl J Med. 2020;383:2219-29.

23. Toth-Manikowski S, Atta MG. Diabetic kidney disease: pathophysiology and therapeutic targets. J Diabetes Res. 2015;2015:697010.

24. Metcalfe W. How does early chronic kidney disease progress? A background paper prepared for the UK Consensus Conference on early chronic kidney disease. Nephrol Dial Transplant. 2007;22:ix26-30.

25. Glassock RJ, Winearls C. Ageing and the glomerular filtration rate: truths and consequences. Trans Am Clin Climatol Assoc. 2009;120:419-28.

26. Lee WC, Lee YT, Li LC, et al. The number of comorbidities predicts renal outcomes in patients with stage 3-5 chronic kidney disease. J Clin Med. 2018;7:493.

27. Jankowski J, Floege J, Fliser D, Bohm M, Marx N. Cardiovascular disease in chronic kidney disease: pathophysiological insights and therapeutic options. Circulation. 2021;143:1157-72.

28. Rangaswami J, Bhalla V, Blair JEA, et al. Cardiorenal syndrome: classification, pathophysiology, diagnosis, and treatment strategies. A scientific statement from the American Heart Association. Circulation. 2019;139:e840-78. 
29. Kazancioglu R. Risk factors for chronic kidney disease: an update. Kidney Int Suppl. 2013;3:368-71.

30. Choi HS, Han KD, Jung JH, et al. The risk of endstage renal disease in systemic lupus erythematosus: a nationwide population-based study in Korea. Medicine (Baltimore). 2019;98:e16420.

31. Wyatt RJ, Julian BA. IgA nephropathy. N Engl J Med. 2013;368:2402-14.

32. Tuttle KR, Brosius FC 3rd, Cavender MA, et al. SGLT2 inhibition for CKD and cardiovascular disease in type 2 diabetes: report of a scientific workshop sponsored by the National Kidney Foundation. Am J Kidney Dis. 2021;77:94-109.

33. de Boer IH, Rue TC, Hall YN, Heagerty PJ, Weiss NS, Himmelfarb J. Temporal trends in the prevalence of diabetic kidney disease in the United States. JAMA. 2011;305:2532-9.

34. Afkarian M, Zelnick LR, Hall YN, et al. Clinical manifestations of kidney disease among US adults with diabetes, 1988-2014. JAMA. 2016;316:602-10.

35. Vallon V, Thomson SC. The tubular hypothesis of nephron filtration and diabetic kidney disease. Nat Rev Nephrol. 2020;16:317-36.

36. Siragy HM, Carey RM. Role of the intrarenal reninangiotensin-aldosterone system in chronic kidney disease. Am J Nephrol. 2010;31:541-50.

37. Yang R, Smolders I, Dupont AG. Blood pressure and renal hemodynamic effects of angiotensin fragments. Hypertens Res. 2011;34:674-83.

38. Alicic RZ, Rooney MT, Tuttle KR. Diabetic kidney disease: challenges, progress, and possibilities. Clin J Am Soc Nephrol. 2017;12:2032-45.

39. Vallon V, Komers R. Pathophysiology of the diabetic kidney. Compr Physiol. 2011;1:1175-232.

40. Yoon SS, Carroll MD, Fryar CD. Hypertension prevalence and control among adults: United States, 2011-2014. NCHS Data Brief. 2015;1-8.

41. Ku E, Lee BJ, Wei J, Weir MR. Hypertension in CKD: core curriculum 2019. Am J Kidney Dis. 2019;74: 120-31.

42. Bidani AK, Griffin KA. Pathophysiology of hypertensive renal damage: implications for therapy. Hypertension. 2004;44:595-601.

43. Bidani AK, Polichnowski AJ, Loutzenhiser R, Griffin KA. Renal microvascular dysfunction, hypertension and CKD progression. Curr Opin Nephrol Hypertens. 2013;22:1-9.
44. US Renal Data System. 2018 Annual Data Report. Volume 1: CKD in the United States. Chapter 4: cardiovascular disease in patients with CKD. Am J Kidney Dis. 2018;73:S79-98.

45. Go AS, Chertow GM, Fan D, McCulloch CE, Hsu CY. Chronic kidney disease and the risks of death, cardiovascular events, and hospitalization. N Engl J Med. 2004;351:1296-305.

46. Levin A, Foley RN. Cardiovascular disease in chronic renal insufficiency. Am J Kidney Dis. 2000;36:S24-30.

47. Valdivielso JM, Rodriguez-Puyol D, Pascual J, et al. Atherosclerosis in chronic kidney disease: more, less, or just different? Arterioscler Thromb Vasc Biol. 2019;39:1938-66.

48. Wang Y, Chen X, Song Y, Caballero B, Cheskin LJ. Association between obesity and kidney disease: a systematic review and meta-analysis. Kidney Int. 2008;73:19-33.

49. Hsu CY, McCulloch CE, Iribarren C, Darbinian J, Go AS. Body mass index and risk for end-stage renal disease. Ann Intern Med. 2006;144:21-8.

50. Chang AR, Grams ME, Navaneethan SD. Bariatric surgery and kidney-related outcomes. Kidney Int Rep. 2017;2:261-70.

51. Mende CW, Einhorn D. Fatty kidney disease: a new renal and endocrine clinical entity? Describing the role of the kidney in obesity, metabolic syndrome, and type 2 diabetes. Endocr Pract. 2019;25:854-8.

52. American Diabetes Association. 6. Glycemic targets: Standards of Medical Care in Diabetes-2021. Diabetes Care. 2021;44:S73-84.

53. Perkovic V, Heerspink HL, Chalmers J, et al. Intensive glucose control improves kidney outcomes in patients with type 2 diabetes. Kidney Int. 2013;83: 517-23.

54. Wong MG, Perkovic V, Chalmers J, et al. Long-term benefits of intensive glucose control for preventing end-stage kidney disease: ADVANCE-ON. Diabetes Care. 2016;39:694-700.

55. SPRINT Research Group, Wright JT Jr, Williamson $\mathrm{JD}$, et al. A randomized trial of intensive versus standard blood-pressure control. N Engl J Med. 2015;373:2103-16.

56. ACCORD Study Group, Cushman WC, Evans GW, et al. Effects of intensive blood-pressure control in type 2 diabetes mellitus. N Engl J Med. 2010;362: 1575-85. 
57. Nadkarni GN, Chauhan K, Rao V, et al. Effect of intensive blood pressure lowering on kidney tubule injury: findings from the ACCORD trial study participants. Am J Kidney Dis. 2019;73:31-8.

58. Whelton PK, Carey RM, Aronow WS, et al. 2017 ACC/AHA/AAPA/ABC/ACPM/AGS/APhA/ASH/ ASPC/NMA/PCNA guideline for the prevention, detection, evaluation, and management of high blood pressure in adults: a report of the American College of Cardiology/American Heart Association Task Force on Clinical Practice Guidelines. J Am Coll Cardiol. 2018;71:e127-248.

59. Gashti CN, Bakris GL. The role of calcium antagonists in chronic kidney disease. Curr Opin Nephrol Hypertens. 2004;13:155-61.

60. Georgianos PI, Agarwal R. Ambulatory blood pressure reduction with SGLT-2 inhibitors: dose-response meta-analysis and comparative evaluation with low-dose hydrochlorothiazide. Diabetes Care. 2019;42:693-700.

61. Martens RJH, Houben A, Kooman JP, et al. Microvascular endothelial dysfunction is associated with albuminuria: the Maastricht Study. J Hypertens. 2018;36:1178-87.

62. Bakris GL, Molitch M. Microalbuminuria as a risk predictor in diabetes: the continuing saga. Diabetes Care. 2014;37:867-75.

63. van der Velde $\mathrm{M}$, Matsushita $\mathrm{K}$, Coresh J, et al. Lower estimated glomerular filtration rate and higher albuminuria are associated with all-cause and cardiovascular mortality. A collaborative metaanalysis of high-risk population cohorts. Kidney Int. 2011;79:1341-52.

64. Heerspink HJ, Kropelin TF, Hoekman J, de Zeeuw D, Reducing Albuminuria as Surrogate Endpoint (REASSURE) Consortium. Drug-induced reduction in albuminuria is associated with subsequent renoprotection: a meta-analysis. J Am Soc Nephrol. 2015;26:2055-64.

65. de Zeeuw D, Remuzzi G, Parving HH, et al. Albuminuria, a therapeutic target for cardiovascular protection in type 2 diabetic patients with nephropathy. Circulation. 2004;110:921-7.

66. Lewis EJ, Hunsicker LG, Bain RP, Rohde RD. The effect of angiotensin-converting-enzyme inhibition on diabetic nephropathy. The Collaborative Study Group. N Engl J Med. 1993;329:1456-62.

67. Jong N, Chertow G, Hou FF, et al. Dapagliflozin decreases albuminuria in patients with chronic kidney disease with and without type 2 diabetes: insights from the DAPA-CKD trial. Nephrol Dial Transplant. 2021;36:48.
68. Tuttle KR, Lakshmanan MC, Rayner B, et al. Dulaglutide versus insulin glargine in patients with type 2 diabetes and moderate-to-severe chronic kidney disease (AWARD-7): a multicentre, open-label, randomised trial. Lancet Diabetes Endocrinol. 2018;6:605-17.

69. Bae JH, Kim S, Park EG, Kim SG, Hahn S, Kim NH. Effects of dipeptidyl peptidase- 4 inhibitors on renal outcomes in patients with type 2 diabetes: a systematic review and meta-analysis. Endocrinol Metab. 2019;34:80-92.

70. Mosenzon O, Leibowitz G, Bhatt DL, et al. Effect of saxagliptin on renal outcomes in the SAVOR-TIMI 53 trial. Diabetes Care. 2017;40:69-76.

71. Centers for Disease Control and Prevention. National diabetes statistics report, 2020. https:// www.cdc.gov/diabetes/pdfs/data/statistics/ national-diabetes-statistics-report.pdf. Accessed 31 May 2020.

72. Hales CM, Carroll MD, Fryar CD, Ogden CL. Prevalence of obesity and severe obesity among adults: United States, 2017-2018. NCHS Data Brief. 2020;1-8.

73. Ejerblad E, Fored CM, Lindblad P, Fryzek J, McLaughlin JK, Nyren O. Obesity and risk for chronic renal failure. J Am Soc Nephrol. 2006;17: 1695-702.

74. Afshinnia F, Wilt TJ, Duval S, Esmaeili A, Ibrahim HN. Weight loss and proteinuria: systematic review of clinical trials and comparative cohorts. Nephrol Dial Transplant. 2010;25:1173-83.

75. Navaneethan SD, Yehnert H, Moustarah F, Schreiber MJ, Schauer PR, Beddhu S. Weight loss interventions in chronic kidney disease: a systematic review and meta-analysis. Clin J Am Soc Nephrol. 2009; 4:1565-74.

76. Baigent C, Landray MJ, Reith C, et al. The effects of lowering LDL cholesterol with simvastatin plus ezetimibe in patients with chronic kidney disease (Study of Heart and Renal Protection): a randomised placebo-controlled trial. Lancet. 2011;377:2181-92.

77. Palmer SC, Navaneethan SD, Craig JC, et al. Metaanalysis: erythropoiesis-stimulating agents in patients with chronic kidney disease. Ann Intern Med. 2010;153:23-33.

78. Di Iorio BR, Bellasi A, Raphael KL, et al. Treatment of metabolic acidosis with sodium bicarbonate delays progression of chronic kidney disease: the UBI Study. J Nephrol. 2019;32:989-1001.

79. Mosenzon O, Wiviott SD, Heerspink HJL, et al. The effect of dapagliflozin on albuminuria in DECLARETIMI 58. Diabetes Care. 2021;44:1805-15. 
80. Wheeler DC, Stefansson BV, Jongs N, et al. Effects of dapagliflozin on major adverse kidney and cardiovascular events in patients with diabetic and nondiabetic chronic kidney disease: a prespecified analysis from the DAPA-CKD trial. Lancet Diabetes Endocrinol. 2021;9:22-31.

81. US Food and Drug Administration. Invokana (canagliflozin) tablets, for oral use [prescribing information]. 2020. https://www.accessdata.fda. gov/drugsatfda_docs/label/2020/204042s034lbl. pdf. Accessed 14 June 2021.

82. US Food and Drug Administration. Farxiga ${ }^{\circledR}$ (dapagliflozin) tablets, for oral use [prescribing information]. 2021. https://www.accessdata.fda.gov/ drugsatfda_docs/label/2021/202293s024lbl.pdf. Accessed 14 June 2021.

83. van Bommel EJM, Muskiet MHA, van Baar MJB, et al. The renal hemodynamic effects of the SGLT2 inhibitor dapagliflozin are caused by postglomerular vasodilatation rather than preglomerular vasoconstriction in metformin-treated patients with type 2 diabetes in the randomized, double-blind RED trial. Kidney Int. 2020;97:202-12.

84. Cherney DZ, Perkins BA, Soleymanlou N, et al. Renal hemodynamic effect of sodium-glucose cotransporter 2 inhibition in patients with type 1 diabetes mellitus. Circulation. 2014;129:587-97.

85. Heerspink HJL, Cherney DZI. Clinical implications of an acute dip in eGFR after SGLT2 inhibitor initiation. Clin J Am Soc Nephrol. 2021;16:1278-80.

86. Kraus BJ, Weir MR, Bakris GL, et al. Characterization and implications of the initial estimated glomerular filtration rate "dip" upon sodium-glucose cotransporter-2 inhibition with empagliflozin in the EMPAREG OUTCOME trial. Kidney Int. 2021;99:750-62.

87. Mistry S, Eschler DC. Euglycemic diabetic ketoacidosis caused by SGLT2 inhibitors and a ketogenic diet: a case series and review of literature. AACE Clin Case Rep. 2021;7:17-9.

88. Heerspink HJ, Cherney D, Postmus D, et al. A prespecified analysis of the Dapagliflozin and Prevention of Adverse Outcomes in Chronic Kidney Disease (DAPA-CKD) randomized controlled trial on the incidence ofabrupt declines in kidney function. Kidney Int. 2021. https://doi.org/10.1016/j.kint. 2021.09.005.

89. US Food and Drug Administration. Jardiance ${ }^{\circledR}$ (empagliflozin) tablets, for oral use [prescribing information]. 2021. https://www.accessdata.fda. gov/drugsatfda_docs/label/2014/204629s000lbl. pdf. Accessed 17 Aug 2021.
90. Zhao M, Sun S, Huang Z, Wang T, Tang H. Network meta-analysis of novel glucose-lowering drugs on risk of acute kidney injury. Clin J Am Soc Nephrol. 2020;16:70-8.

91. Wilcox CS. Antihypertensive and renal mechanisms of SGLT2 (sodium-glucose linked transporter 2) inhibitors. Hypertension. 2020;75:894-901.

92. Cianciolo G, De Pascalis A, Capelli I, et al. Mineral and electrolyte disorders with SGLT2i therapy. JBMR Plus. 2019;3:e10242.

93. Perkovic V, Jardine MJ, Neal B, et al. Canagliflozin and renal outcomes in type 2 diabetes and nephropathy [supplemental appendix]. N Engl J Med. 2019;380:2295-306. https://doi.org/10.1056/ NEJMoa1811744/suppl_file/nejmoa_appendix.pdf. Accessed 5 Oct 2021.

94. Ye N, Jardine MJ, Oshima M, et al. Blood pressure effects of canagliflozin and clinical outcomes in type 2 diabetes and chronic kidney disease: insights from the CREDENCE Trial. Circulation. 2021;143: 1735-49.

95. Herat LY, Magno AL, Rudnicka C, et al. SGLT2 inhibitor-induced sympathoinhibition: a novel mechanism for cardiorenal protection. JACC Basic Transl Sci. 2020;5:169-79.

96. Neal B, Perkovic V, Mahaffey KW, et al. Canagliflozin and cardiovascular and renal events in type 2 diabetes. N Engl J Med. 2017;377:644-57.

97. McGovern AP, Hogg M, Shields BM, et al. Risk factors for genital infections in people initiating SGLT2 inhibitors and their impact on discontinuation. BMJ Open Diabetes Res Care. 2020;8:e001238.

98. US Food and Drug Administration. Kerendia (finerenone) tablets, for oral use [prescribing information]. 2021. https://www.accessdata.fda.gov/ drugsatfda_docs/label/2021/215341s000lbl.pdf. Accessed 17 Aug 2021.

99. Gerstein HC, Colhoun HM, Dagenais GR, et al. Dulaglutide and renal outcomes in type 2 diabetes: an exploratory analysis of the REWIND randomised, placebo-controlled trial. Lancet. 2019;394:131-8.

100. Mann JFE, Ørsted DD, Brown-Frandsen K, et al. Liraglutide and renal outcomes in type 2 diabetes. N Engl J Med. 2017;377:839-48.

101. Muskiet MHA, Tonneijck L, Huang Y, et al. Lixisenatide and renal outcomes in patients with type 2 diabetes and acute coronary syndrome: an exploratory analysis of the ELIXA randomised, placebo-controlled trial. Lancet Diabetes Endocrinol. 2018;6:859-69. 